Chiara Bellia, Martina Zaninotto, Chiara Cosma, Luisa Agnello, Bruna Lo Sasso, Giulia Bivona, Mario Plebani ${ }^{\mathrm{a}}$ and Marcello Ciaccio ${ }^{\mathrm{a}, \star}$

\title{
Definition of the upper reference limit of glycated albumin in blood donors from Italy
}

https://doi.org/10.1515/cclm-2017-0179

Received March 1, 2017; accepted April 24, 2017; previously published online June 6, 2017

\section{Abstract}

Background: Glycated Albumin (GA) has been proposed as a short-term indicator of glycemic homeostasis. The aim of this study is to describe the distribution of GA in a large sample of blood donors from Italy to evaluate whether demographic features, namely age and sex, could influence GA levels and define specific reference limits.

Methods: The study included 1334 Italian blood donors. GA was measured using an enzymatic method (quantILab Glycated Albumin, IL Werfen, Germany). The upper reference limit (URL) was calculated using the non-parametric percentile method.

Results: A modest, although significant, increase of GA was observed in relation to age $(\mathrm{p}<0.001)$, especially in males, where the differences were more pronounced $(\mathrm{p}<0.001$ in males, $\mathrm{p}=0.003$ in females). Slight differences were documented based on sex (12\% [11.3-12.8] in males; $12.2 \%$ [11.4-13.1] in females; $\mathrm{p}=0.01)$. After excluding individuals with fasting plasma glucose $\geq 7 \mathrm{mmol} / \mathrm{L}$, the calculated GA URL was $14.5 \%$ (95\% CI: 14.3-14.7). Subjects with GA $>14.5 \%$ presented a mean age of $48.4 \pm 12.2$ years, $66.7 \%$ were males and the mean glucose was $6.88 \pm 2.5 \mathrm{mmol} / \mathrm{L}$.

Conclusions: GA in Caucasians shows a similar increasing trend at older ages documented in other ethnicities. The

\footnotetext{
aMario Plebani and Marcello Ciaccio are both considered last authors. *Corresponding author: Marcello Ciaccio, MD, PhD, Department of Biopathology and Medical Biotechnologies, Section of Clinical Biochemistry and Clinical Molecular Medicine, University of Palermo, Via del Vespro, 129, 90127 Palermo, Italy, Phone: +39 091 6553296, Fax +39091655 3275, E-mail: marcello.ciaccio@unipa.it; and Department of Laboratory Medicine, University-Hospital, Palermo, Italy Chiara Bellia, Luisa Agnello, Bruna Lo Sasso and Giulia Bivona: Department of Biopathology and Medical Biotechnologies, Section of Clinical Biochemistry and Clinical Molecular Medicine, University of Palermo, Palermo, Italy

Martina Zaninotto, Chiara Cosma and Mario Plebani: Department of Laboratory Medicine, University-Hospital, Padova, Italy. http://orcid. org/0000-0002-0270-1711 (M. Plebani)
}

definition of the URL in this population could be useful for both clinical studies, which will clarify the role of GA for diagnosing and monitoring diabetes, and will encourage the introduction of GA in clinical practice.

Keywords: blood donors; diabetes; glycated albumin; reference limit.

\section{Introduction}

Diabetes has become a public health challenge in recent years given its increasing prevalence worldwide [1]. Universally-adopted diagnostic criteria include the increase of fasting plasma glucose (FPG), 2-h plasma glucose after an oral glucose tolerance tests and $\mathrm{HbA}_{1 c}$ [2], which are considered good indicators of the metabolic imbalance for diabetes mellitus. Monitoring glycemic control represents the basis for managing diabetic patients, and it is achieved by measuring $\mathrm{HbA}_{1 \mathrm{c}}$ and self-monitoring blood glucose [3]. $\mathrm{HbA}_{\mathrm{Ac}}$ is a product of the glycation process that potentially involves all proteins, although at different rates based on cellular localization, circulating levels and amino acid composition. Although $\mathrm{HbA}_{1 c}$ is widely used for both diagnosis and monitoring diabetes, its clinical use is debated in some settings, such as pregnancy, anemia, frequent transfusions, end-stage renal disease. Moreover, given the lifespan of erythrocytes, it retrospectively reflects glycemic levels in the previous 60-90 days, but its diagnostic value is limited when considering shorter intervals.

Although achieving a good glycemic control by maintaining $\mathrm{HbA}_{1 \mathrm{c}}$ levels $<7 \%$ ( $53 \mathrm{mmol} / \mathrm{mol}$ ) remains a primary goal for diabetic patients, further evidence suggests that limiting glycemic variations, in terms of both hyper- and hypoglycemic peaks, impacts cardiovascular outcomes more than maintaining constant, although high, plasma glucose [4]. Interestingly, some authors speculated that a short-term indicator of protein glycation could reflect glycemic fluctuations better than $\mathrm{HbA}_{1 c}$, although this hypothesis still requires confirmation from large prospective studies [5-7].

Glycated albumin (GA) has been proposed as a short-term indicator of glycemic homeostasis, given that albumin turnover is about 20 days [8]. Recently, a 
colorimetric-enzymatic method has been developed to assess circulating levels of GA on automated analyzers, commonly used for the measurement of clinical chemistry traditional test $[9,10]$. GA has been introduced in Japan for blood donor screenings as an effective indicator for individuals at risk of developing diabetes [11]. Results from the Atherosclerosis Risk in Communities (ARIC) study demonstrated that GA was associated to the risk of developing diabetes independently of $\mathrm{HbA}_{1 \mathrm{c}}$ and fasting glucose [12]. A large community-based study conducted in China evaluated the correlation between GA with FPG and $\mathrm{HbA}_{1 \mathrm{c}}$, and proposed the GA cut-off at $15.5 \%$ to identify a poor glycemic control in clinical practice [13]. Nevertheless, no studies analyzing the reference values of GA have been published for a sample of the Caucasian population. The aim of this study is to describe the distribution of GA in a large sample of blood donors from Italy to evaluate whether demographic features, namely age and sex, could influence GA levels and to define specific reference limits.

\section{Materials and methods}

\section{Subjects}

The study included 1356 consecutive donors between the ages of 18 and 65 years, at the Unit of Transfusion Medicine of Villa SofiaCervello Hospital in Palermo from April 2016 to September 2016. The study protocol was approved by the Local Ethics Committee in accordance with the Declaration of Helsinki, and all study participants gave informed consent. After excluding subjects with no FPG available $(n=22)$, the remaining 1334 were considered for this study. The mean age of the entire study group was $41.4 \pm 12.2$ years, $70.5 \%$ were males. The exclusion criteria for donation were: neoplasia; autoimmune diseases; celiac disease; coronary artery disease; angina; cardiac arrhythmias; history of cerebrovascular disease; arterial thrombosis; recurrent deep vein thrombosis; hypertension with organ damage; organic diseases of the central nervous system (CNS); transplant recipients; coagulopathies; epilepsy; anaphylaxis; infectious diseases; any chronic hepatic, gastrointestinal, urogenital, hematologic, immunologic, renal, metabolic and respiratory disease. In accordance with local laws, diabetics with a good glycemic control and no insulin treatment were admitted for donation. Glycemic control was evaluated basing on self-reported $\mathrm{HbA}_{1 \mathrm{c}}<53 \mathrm{mmol} / \mathrm{mol}$; preprandial plasma glucose between 4.4 and $7.2 \mathrm{mmol} / \mathrm{L}$; and postprandial plasma glucose $<10 \mathrm{mmol} / \mathrm{L}$. All subjects were Italian, except for two individuals from Africa and one from Asia.

The subjects included in this study were further classified as normal, as having impaired fasting glucose (IFG) and diabetics based on FPG. Particularly, subjects with FPG $\leq 5.5 \mathrm{mmol} / \mathrm{L}$ were classified as normal; subjects with FPG from $5.6 \mathrm{mmol} / \mathrm{L}$ to $6.9 \mathrm{mmol} / \mathrm{L}$ were classified as having IFG; subjects with FPG $\geq 7.0 \mathrm{mmol} / \mathrm{L}$ were classified as diabetics. Blood samples, complete medical history and written informed consent were collected from participants upon arrival at the Transfusion Medicine Unit.

\section{Laboratory analysis}

FPG was measured immediately using the Architect C800 instrument and reagents (Abbott). Plasma K2-EDTA (Greiner Bio-One) was aliquoted and stored at $-80^{\circ}$ until GA analysis. GA was measured using an enzymatic method (quantILab Glycated Albumin, IL Werfen, German) on ILab Taurus system (IL Werfen). This assay measures total albumin and the glycated amino acids originated from the proteolytic degradation of glycated albumin simultaneously. GA concentration is reported as a percentage of the total albumin, and corrected with the inter-method arithmetic expression designed to adhere to the GA levels determined with HPLC [10]. Imprecision of the assay was evaluated analyzing two samples, at the concentrations of $15.2 \%$ and $32.1 \%$, respectively, 20 times in the same run (within-run CV \%), and repeated for 15 days (between-run CV \%). Between-run CV \% were 3.1 and 2.5 at low and high concentrations, respectively. Within-run CV \% were $1.3 \%$ and $0.9 \%$ at low and high concentrations, respectively.

\section{Statistical analysis}

All statistical analysis was performed using SPSS software. Variables were presented as mean \pm standard deviation (SD) for continuous variables, as medians with interquartile ranges (IQR) for not-normally distributed continuous variables, and as a percentage for the categorical variables. Normality of distributions was assessed using the Kolmogorov-Smirnov test. Differences between normally-distributed variables were evaluated using ANOVA. Differences in GA concentration among age- and sex groups were evaluated by the Kruskal-Wallis test. For upper reference limit (URL) determination, subjects with FPG $\geq 7.0 \mathrm{mmol} / \mathrm{L}(\mathrm{n}=26)$ were excluded for the analysis, based on the American Diabetes Association guidelines for diabetes diagnosis [2]. URL was calculated with the non-parametric percentile method in accordance with the Clinical and Laboratory Standard Institute CLSI C28-A3, considering values in the $95 \%$ of the distribution [14]. The statistical significance of $\mathrm{p}<0.05$ was accepted for all the tests.

\section{Results}

Initially, this study included 1356 individuals. Twenty-two subjects were excluded because FPG was not available, so the analysis was performed on the remaining 1334 subjects, of which $941(70.5 \%)$ were males. The mean age of the study group was $41.4 \pm 12.2$ years.

Table 1 shows the distribution of plasma GA in relation to age. Interestingly, a modest, although significant, increase of GA with age was observed ( $p<0.001)$. When considering GA levels based on sex, we found that GA in males (12\%; IQR: 11.3-12.8) was slightly lower than in females (12.2\%; IQR: 11.4-13.1) $(\mathrm{p}=0.01)$. When males and females were stratified by age, GA showed a different 
Table 1: Plasma GA values (median and interquartile range), based on age. Plasma GA was differently distributed in age classes $(\mathrm{p}<0.001)$

\begin{tabular}{lrr}
\hline Age range, years & $\mathbf{n}(\%)$ & GA, \% \\
\hline $18-30$ & $304(22.8)$ & $11.8(11.2-12.5)$ \\
$31-40$ & $273(20.5)$ & $12(11.2-12.7)$ \\
$41-50$ & $417(31.2)$ & $12.2(11.4-12.8)$ \\
$51-65$ & $340(25.5)$ & $12.4(11.7-13.3)$ \\
Total & $1334(100)$ & $12.1(11.4-12.8)$ \\
\hline
\end{tabular}

distribution based on age in males and, to a lesser extent, in females (Table 2). Specifically, GA shows a constant increasing trend in males from 18 to 65 years old, while a similar trend cannot be observed in females (Figure 1).

After excluding 26 individuals with $\mathrm{FPG} \geq 7.0 \mathrm{mmol} / \mathrm{L}$, the remaining 1308 were considered for the calculation of the URL of GA, defined as the $97.5 \%$ of the distribution. In this sample, the GA URL was $14.5 \%$ (95\% CI: 14.3-14.7). When considering males and females separately, the URL was 14.4\% (95\% CI: 14.1-14.7) in males and 14.6\% (95\% CI: 14.5-15.2) in females.

Subjects were classified as normal ( $\mathrm{FPG} \leq 5.5 \mathrm{mmol} / \mathrm{L}$ ), impaired fasting glucose (IFG) (FPG from $5.6 \mathrm{mmol} / \mathrm{L}$ to $6.9 \mathrm{mmol} / \mathrm{L}$ ), and diabetics ( $\mathrm{FPG} \geq 7.0 \mathrm{mmol} / \mathrm{L}$ ) based on FPG. As shown in Table 3, GA was higher in diabetics (14.6\%; IQR: $12.5-18.9)$ in comparison to subjects with IFG (12.5\%; IQR: 11.6-13.3) and normal subjects (12\%; IQR: 11.3-12.8) $(\mathrm{p}<0.001)$. As expected, also age and FPG showed the same trend $(p<0.001$ and $p=0.003$, respectively). Particularly, FPG was $4.8 \pm 0.4 \mathrm{mmol} / \mathrm{L}$ in normal; $5.9 \pm 0.4 \mathrm{mmol} / \mathrm{L}$ in IFG and $8.8 \pm 2.1 \mathrm{mmol} / \mathrm{L}$ in diabetic subjects, respectively. Moreover, $1.3 \%$ of normal and $7.8 \%$ of IFG subjects had high GA.

Table 2: Plasma GA distribution in males (A) and females (B) based on age. Plasma GA is expressed as median and interquartile range.

\begin{tabular}{lrr}
\hline Age range, years & $\mathbf{n}(\%)$ & GA, \% \\
\hline Males & & \\
$18-30$ & $170(55.9)$ & $11.6(11.1-12.5)$ \\
$31-40$ & $205(75)$ & $11.9(11.1-12.5)$ \\
$41-50$ & $315(75.5)$ & $12.1(11.4-12.8)$ \\
$51-65$ & $251(73.8)$ & $12.4(11.7-13.2)$ \\
Total & $941(70.5)$ & $12(11.3-12.8)$ \\
Females & & \\
$18-30$ & $134(44.1)$ & $12(11.2-12.5)$ \\
$31-40$ & $68(25)$ & $12.5(11.6-13.3)$ \\
$41-50$ & $102(24.5)$ & $12.4(11.5-13.3)$ \\
$51-65$ & $89(26.2)$ & $12.4(11.4-13.3)$ \\
Total & $393(29.5)$ & $12.2(11.4-13.1)$ \\
\hline
\end{tabular}

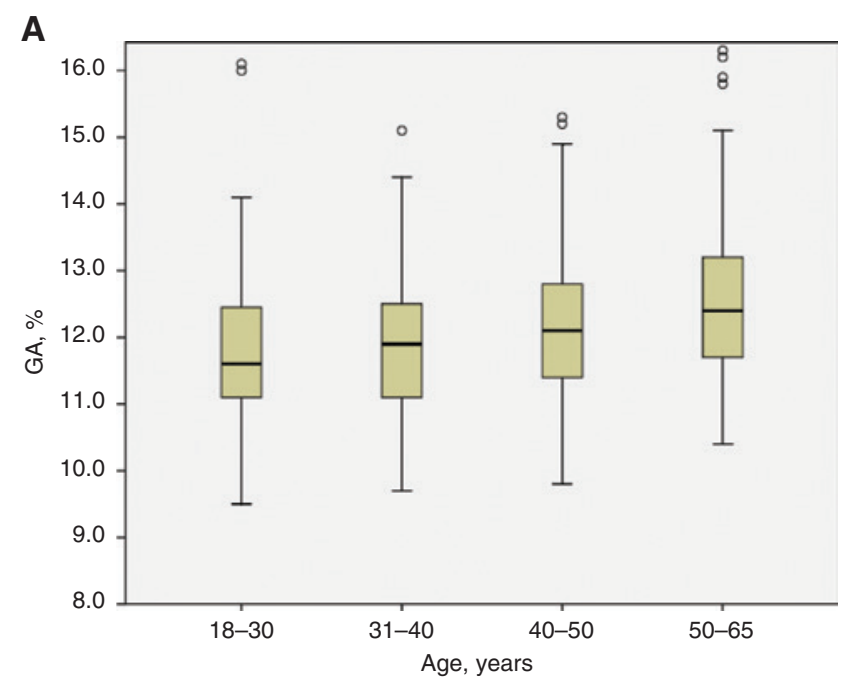

B

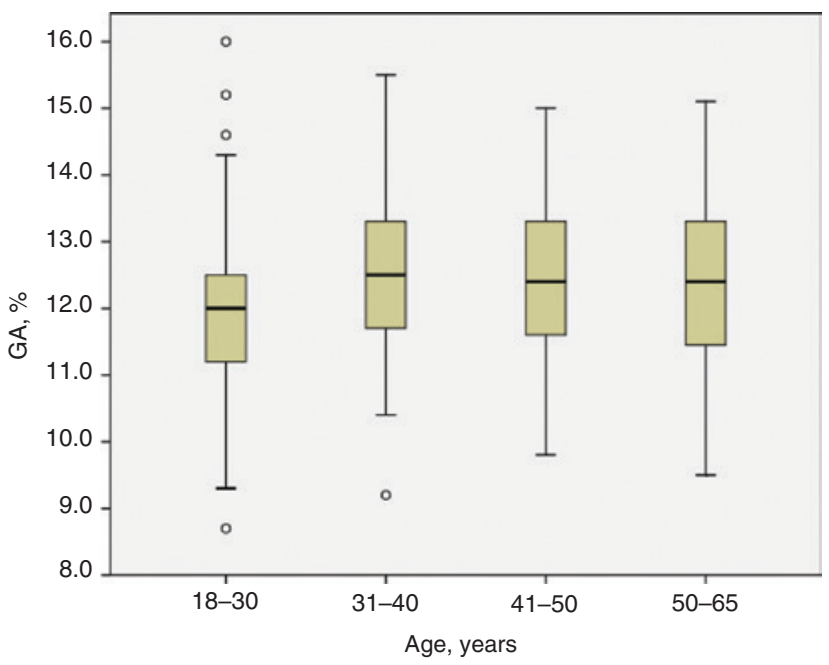

Figure 1: GA shows a constant increasing trend in males, while a similar trend cannot be observed in females.

Plasma GA distribution based on age in males (A) and in females (B).

Interestingly, when using the GA cut-off at $14.5 \%$, calculated as the $97.5^{\circ}$ percentile of the GA distribution as previously described, 3.1\% (42/1334) of donors were classified as having high GA, while only $1.9 \%$ (26/1334) were diabetics based on FPG, suggesting that the two markers could reflect different aspects of glucose homeostasis. Overall, subjects with $\mathrm{GA} \geq 14.5 \%$ presented a mean age of $48.4 \pm 12.2$ years, $66.7 \%$ were males and the mean FPG was $6.88 \pm 2.5 \mathrm{mmol} / \mathrm{L}$.

\section{Discussion}

GA can be considered a short-term indicator of glucose homeostasis that could help evaluate glycemic control. 
Table 3: FPG and GA levels distribution based on ADA guidelines for diabetes mellitus diagnosis [2].

\begin{tabular}{|c|c|c|c|c|}
\hline & Normal $(n=1154)$ & IFG $(n=154)$ & Diabetes $(n=26)$ & p-Value \\
\hline Age, years & $40 \pm 12.2$ & $48 \pm 10.5$ & $49.6 \pm 9$ & $<0.001$ \\
\hline Sex, males & $68.8 \%$ & $80.6 \%$ & $76.9 \%$ & 0.007 \\
\hline $\mathrm{FPG}, \mathrm{mmol} / \mathrm{L}$ & $4.8 \pm 0.4$ & $5.9 \pm 0.4$ & $8.8 \pm 2.1$ & 0.003 \\
\hline $\mathrm{GA}, \%$ & $12(11.3-12.8)$ & $12.5(11.6-13.3)$ & $14.6(12.5-18.9)$ & $<0.001$ \\
\hline GA positive, $\mathrm{n}(\%)$ & $16(1.3)$ & $12(7.8)$ & $13(50)$ & $<0.001$ \\
\hline
\end{tabular}

The GA positivity was based on the URL of $14.5 \%$, namely the $97.5^{\circ}$ percentile of its distribution in this study.

Its clinical use has been proposed in specific settings in which $\mathrm{HbA}_{1 c}$, recognized by several international guidelines as the gold standard for monitoring diabetes, is affected by major limitations, such as in anemia or shortterm control of anti-diabetic therapy. Moreover, an early diagnosis of diabetes, when the metabolic imbalance is still moderate, remains a challenge. Clinical research on the role of GA in diagnosing and monitoring diabetes requires the knowledge of its circulating levels in healthy individuals, as well as the standardization of analytical methods. The use of an enzymatic-colorimetric assay allows the high-throughput measurement of GA because it is performed on the same sample used for routine clinical chemistry tests, and with analyzers commonly used in clinical laboratories. The standardization of the method for $\mathrm{GA}$ and the definition of biological variability have been recently achieved [10, 15]; however, no large studies on a reference Caucasian population have been published yet. Moreover, before introducing GA in clinical practice, it is important to define specific reference limits and the potential influence of age and sex on its distribution in a reference population.

In this study, a large sample of blood donors was considered for assessing the influence of sex and age on circulating GA levels, and for GA URL calculation. Results showed a slight increase of GA with age, particularly in males. The increase of GA with age could be explained by the well-documented imbalance of glucose homeostasis at older ages [16]. Our study was designed in aim to define the URL in a reference population, in which physiological and pathological conditions that could interfere with analyte concentration are controlled as much as possible. For this reason we chose blood donors to conduct our study, and not individuals from the general population, because in the latter controlling these variables is challenging.

Our findings agree with Araki et al., who showed a similar trend for GA at increasing ages in a large study on blood donors conducted in Japan [11]. In our study, we did not find a clinically relevant increase of GA in females at older ages, probably due to the different impact of ageing on glucose homeostasis among men and women [17]. It should be noted that in our study females were less represented than in the study by Araki et al., affecting the statistical value of the analysis.

Although the similar increasing trend of GA with age, the GA median levels documented in the study by Araki et al. were higher than the ones found in our study, probably due to the different ethnicity. It can be hypothesized that common allelic variants could be responsible of the expression of different albumin amino acid sequences among populations and, consequently, have different glycation patterns. In addition to genetic background, several other factors could explain different glycation rates among a population. For example, it has been demonstrated in vitro that the structure of the side chain of Lys525, the most frequent site of glycation, is altered in presence of fatty acids [18]. It is reasonable that in vivo different concentrations of free fatty acids, probably due to different dietary habits between Asian and Western populations, could affect glycation rate of such residue by altering the surrounding structure [19]. Notably, our population, consisting of only Caucasian subjects, was highly homogeneous for ethnicity. In a large cohort study involving 1575 individuals from Japanese general population (Kyushu and Okinawa Population Study, KOPS), no significant differences for GA levels were detected in different sexes [13]. This apparent discrepancy with our result can be explained considering that the mean age of the population considered in the KOPS study was 49.9 years, significantly higher than the one observed in our population, and that the main sex differences we observed were at younger ages, becoming no more appreciable at the higher ones.

After excluding subjects with diabetes, based on their FPG, we reported the URL of GA as $14.5 \%$. Although we found a statistically significant association of GA with ageing in the entire study sample, and especially in males, where the differences were more pronounced (GA levels varies from $11.6 \%$ in younger males to $12.4 \%$ in the older ones), we decided to consider a unique URL because further stratification for age beyond sex would have yielded to small subgroups and, thus, have limited the 
statistic value of the analysis. Moreover, although statistically significant, the age-dependent differences in males can be considered of minor clinical relevance. However, it requires confirmation from studies assessing the clinical use of GA in well-characterized diabetic patients and subjects at risk of developing diabetes.

Considering the GA URL of $14.5 \%$, namely the $97.5^{\circ}$ percentile of its distribution in this study, the prevalence of high GA was 4.4\%. In a similar study, Araki et al. [11] found that subjects with altered glucose metabolism and diabetes, defined as $\mathrm{GA}>16.5 \%$ and classified in the same group, were $2.8 \%$ of males and $2.3 \%$ of females. Although results are not directly comparable, they could suggest a different prevalence of elevated GA in the two populations.

Few studies have reported the reference range of GA among the Caucasian population. In the study by Testa et al. higher values of GA were reported in a group of 252 European subjects [20]. This difference may be due to a less strict selection of individuals. In our study, indeed, subjects affected by any chronic hepatic, malignant, kidney, thyroid disorders that could affect albumin concentration, were excluded.

Interestingly, in this study a significant proportion of subjects classified as normal and IFG based on their FPG, $1.3 \%$ and $7.8 \%$, respectively, had high levels of GA. This result indicates that, if confirmed in clinical studies, GA could identify a significant proportion of subjects with an altered glucose homeostasis that FPG couldn't identify. Nevertheless, a full comprehension of the mechanisms that link glucose dysmetabolism, albumin glycation and its stability in circulation, is mandatory to confirm this hypothesis. It is known that FPG and GA reflect different aspects of metabolic control, the first being an immediate indicator, and the second a short-term retrospective indicator. Moreover, GA reflects both postprandial and fasting plasma glucose. For these reasons, it is understandable that the two markers have different diagnostic performances. A limitation of the study is that a strictly adherent classification to ADA criteria of normal, IGT and diabetics is lacking because $\mathrm{HbA}_{1 c}$ and OGTT were not available.

In conclusion, this is the first study describing GA plasma levels in a large sample of blood donors in Italy, and the role of influencing factors such as age and sex. The definition of the URL for GA could be useful for both clinical studies that will clarify the role of GA in diabetes diagnosis and monitoring, and will encourage the introduction of GA in clinical practice.

Acknowledgments: IL Werfen provided the reagents for GA measurement. The company did not play a role in the study design, collection, analysis and interpretation of data, writing of the manuscript, or in the decision to submit the manuscript for publication.

The authors thank Maddalena Muratore of the Unit of Transfusion Medicine of Villa Sofia-Cervello Hospital and Alessia Pivetti of the Department of Biopathology and Medical Biotechnologies, University of Palermo, for contributing to data collection.

Author contributions: All the authors have accepted responsibility for the entire content of this submitted manuscript and approved submission.

Research funding: None declared.

Employment or leadership: None declared.

Honorarium: None declared.

Competing interests: The funding organization(s) played no role in the study design; in the collection, analysis and interpretation of data; in the writing of the report; or in the decision to submit the report for publication.

\section{References}

1. Zimmet P, Alberti KG, Shaw J. Global and societal implications of the diabetes epidemic. Nature 2001;414:782-7.

2. American Diabetes Association. Classification and diagnosis of diabetes. Diabetes Care 2017;40:S11-24.

3. American Diabetes Association. Glycemic targets. Diabetes Care 2017;40:S48-56.

4. Ceriello A, Genovese S, Bosi E. The evolving frontier of diabetes therapy: the renaissance of glycemology. Diabetes Res Clin Pract 2016;118:168-71.

5. Yoshiuchi K, Matsuhisa M, Katakami N, Nakatani Y, Sakamoto $\mathrm{K}$, Matsuoka T, et al. Glycated albumin is a better indicator for glucose excursion than glycated hemoglobin in type 1 and type 2 diabetes. Endocr J 2008;55:503-7.

6. Koga M, Murai J, Morita S, Saito H, Kasayama S. Comparison of annual variability in $\mathrm{HbA}_{1 \mathrm{c}}$ and glycated albumin in patients with type 1 vs. type 2 diabetes mellitus. J Diabetes Complications 2013;27:211-3.

7. Fukami K, Shibata R, Nakayama H, Yamada K, Okuda S, Koga $M$. Serum albumin-adjusted glycated albumin reflects glycemic excursion in diabetic patients with severe chronic kidney disease not treated with dialysis. J Diabetes Complications 2015;29:913-7.

8. Danese E, Montagnana M, Nouvenne A, Lippi G. Advantages and pitfalls of fructosamine and glycated albumin in the diagnosis and treatment of diabetes. J Diabetes Sci Technol 2015;9:169-76.

9. Paroni R, Ceriotti F, Galanello R, Battista Leoni G, Panico A, Scurati $E$, et al. Performance characteristics and clinical utility of an enzymatic method for the measurement of glycated albumin in plasma. Clin Biochem 2007;40:1398-405.

10. Takei I, Hoshino T, Tominaga M, Ishibashi M, Kuwa K, Umemoto $\mathrm{M}$, et al. Committee on Diabetes Mellitus Indices of the Japan Society of Clinical Chemistry-recommended reference measurement procedure and reference materials for glycated albumin determination. Ann Clin Biochem 2016;53(Pt 1):124-32. 
11. Araki T, Ishikawa Y, Okazaki H, Tani Y, Toyooka S, Satake M, et al. Introduction of glycated albumin measurement for all blood donors and the prevalence of a high glycated albumin level in Japan. J Diabetes Investig 2012;3:492-7.

12. Selvin E, Rawlings AM, Grams M, Klein R, Sharrett AR, Steffes M, et al. Fructosamine and glycated albumin for risk stratification and prediction of incident diabetes and microvascular complications: a prospective cohort analysis of the Atherosclerosis Risk in Communities (ARIC) study. Lancet Diabetes Endocrinol 2014;2:279-88.

13. Furusyo N, Koga T, Ai M, Otokozawa S, Kohzuma T, Ikezaki H, et al. Utility of glycated albumin for the diagnosis of diabetes mellitus in a Japanese population study: results from the Kyushu and Okinawa Population Study (KOPS). Diabetologia 2011;54:3028-36.

14. CLSI. Defining, establishing, and verifying reference intervals in the clinical laboratory; approved guideline, $3 \mathrm{rd}$ ed. CLSI document c28-a3. Wayne, PA: Clinical and Laboratory Standards Institute, 2008
15. Montagnana M1, Paleari R, Danese E, Salvagno GL, Lippi G, Guidi GC, et al. Evaluation of biological variation of glycated albumin (GA) and fructosamine in healthy subjects. Clin Chim Acta 2013;423:1-4.

16. Chang AM, Halter JB. Aging and insulin secretion. Am J Physiol Endocrinol Metab 2003;284:E7-12.

17. DECODE Study Group. Age- and sex-specific prevalences of diabetes and impaired glucose regulation in 13 European cohorts. Diabetes Care 2003;26:61-9.

18. Simard JR, Zunszain PA, Ha CE, Yang JS, Bhagavan NV, Petitpas I, et al. Locating high-affinity fatty acid-binding sites on albumin by $x$-ray crystallography and NMR spectroscopy. Proc Natl Acad Sci USA 2005;102:17958-63.

19. Ueda Y, Matsumoto H. Recent topics in chemical and clinical research on glycated albumin. J Diabetes Sci Technol 2015;9:177-82.

20. Testa R, Ceriotti F, Guerra E, Bonfigli AR, Boemi M, Cucchi M, et al. Glycated albumin: correlation to $\mathrm{HbA}_{1 \mathrm{c}}$ and preliminary reference interval evaluation. Clin Chem Lab Med 2017;55:e31-3. 